\title{
Abstracts of Award-Winning Posters, 14th Annual Health Sciences Poster Conference, Faculty of Medicine, Health Sciences Centre, Kuwait University, Kuwait, April 21-23, 2009
}

\section{Dr. Nael Al-Naqeeb Undergraduate Research Award}

\footnotetext{
Expression of Nucleoside Transporters in the Rat Heart: Effects of Streptozotocin-Induced Diabetes

S. Omar, A. Al-Turki, S. Malatiali, Z. Redzic

Department of Physiology, Faculty of Medicine, Kuwait University, Kuwait
}

Introduction: It has been shown in the rat that insulin affects expression of the rat equilibrative nucleoside transporter (rENT) 2 and rat concentrative nucleoside transporter ( $\mathrm{rCNT}$ ) 1 and 2 in cardiac fibroblasts and adenosine uptake by cardiac myocytes. The aim of this study was to explore the effects of streptozotocin (STZ)-induced diabetes in the rat heart on the expression of rENT1 and 2 and rCNT1, 2 and 3. Methods: Diabetes was induced in Sprague-Dawley rats by an i.p. injection of STZ $(60 \mathrm{mg} / \mathrm{kg})$; controls were treated with vehicle. Diabetes was confirmed by plasma glucose $>17 \mathrm{~mm}$. Rats were sacrificed after 4 weeks, and cardiac muscle samples were frozen in liquid $\mathrm{N}_{2}$. Real-time PCR was used to estimate the threshold cycles for target amplification (Ct) values. The differences between the Ct values for ENTs and $\mathrm{CNT}$ and the $\mathrm{Ct}$ values for the housekeeping gene $\beta$-actin were calculated $(\Delta \mathrm{Ct})$, and the difference between diabetic and control rats was tested for significance. Data are presented as mean \pm SD from 3 samples. Results: The Ct value of $\beta$-actin did not differ significantly between the groups $(\mathrm{p}>0.05)$, so this gene was used as the endogenous control. In the control group, the mRNA for rCNT2, rENT2, rENT1 and rCNT1 were detected with the $\Delta \mathrm{Ct}$
$2.4 \pm 0.4,4.3 \pm 0.3,7.4 \pm 0.6$ and $8.1 \pm 0.1$, respectively. The mRNA for rCNT3 was apparently absent. Four-week diabetes caused a significant decrease in the amount of mRNA for rCNT1 ( $p<0.05$ vs. control), while the amount of mRNA for other transporters did not change significantly. Conclusions: The observed changes in the amount of rCNT1 mRNA may indicate a decrease in the amount of this pyrimidine-selective transporter in the membrane, which in turn could reduce the cellular uptake of pyrimidines in diabetes.

\section{Best Postgraduate Awards}

\section{Basic Sciences (MSc)}

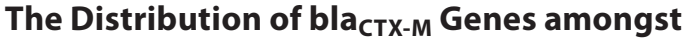 Clinically Significant Escherichia coli Isolates in Eight Major Hospitals in Kuwait \\ Ghayda Al Hashem, N. Al-Sweih, Wafaa Jamal, V.O. Rotimi \\ Department of Microbiology, Faculty of Medicine, Kuwait University, Kuwait}

Introduction: Dissemination of CTX-M extended-spectrum $\beta$-lactamases (ESBL) is pandemic. The production of this enzyme is mediated by the bla СтХ-м gene, which confers resistance to third-generation cephalosporins mainly in Escherichia coli and

\section{KARGER}

Fax +41613061234

E-Mail karger@karger.ch

www.karger.com
(C) 2009 S. Karger AG, Basel

$1011-7571 / 09 / 0186-0493 \$ 26.00 / 0$

Accessible online at:

www.karger.com/mpp 
Klebsiella spp. This study was designed to investigate the distribution of bla ment hospitals. Methods: A total of 876 consecutive isolates were collected from these hospitals, at least 100 per hospital. Production of ESBL was detected by the ESBL Etest method and confirmed by PCR. DNA extracts of ESBL-positive isolates were screened for the presence of the bla $\mathrm{C}_{\mathrm{CTX}-\mathrm{M}}$ gene using the following primers: MA-1 5'-SCS ATG TGC AGY ACC AGT AA-3' and MA-2 5'-CCG CRA TAT GRT TGG TGG TG-3'. Strains with PCR amplicons positive for bla $\mathrm{CTX}_{\mathrm{M}}$ were sequenced using Bigdye Terminator ${ }^{\circledR}$. The nucleotides were then analyzed with freely available software (www.ncbi.nlm.nih.gov/blast). The CTX-M15-positive strains were evaluated for genetic relatedness using pulsed field gel electrophoresis (PFGE) with $\mathrm{XbaI}$ digestion of the genomic DNA. Results: Of the 876 isolates, 113 (12.9\%) were ESBL producers, $88(78 \%)$ of which produced CTX-M ESBL. Among these, CTX-M-15 accounted for $84 \%$, followed by CTX-M-14 (7\%), CTX-M-14b (6\%) and TOHO-1 (3\%). The highest number of E.

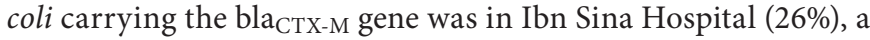
specialist hospital for immunocompromised patients, and the lowest number was found in a maternity hospital (6\%). CTX-M-15 was the predominant CTX-M type in all hospitals, representing 74 (84\%) of the CTX-M ESBL. Of these, 66 (89\%) were found in isolates from Kuwaitis, Egyptians and Indians. The PFGE pattern of the CTX-M-15-positive isolates was heterogeneous. Conclusions: Our data show an explosive emergence of CTX-M-15 ESBL that appears to be evenly distributed in E. coli strains in patients belonging to the predominant nationalities in Kuwait and that demonstrates no evidence of clonal spread.

\section{Resident}

\section{Assessment of Adiposity in Routine Practice - Comparison of Bioelectrical Impedance Analysis of Body Fat Composition with Body Mass Index and Other Anthropometric Measurements}

\author{
H.A.M.Najjar ${ }^{\text {a }}$, N.R.Al-Tajalli ${ }^{\text {a }}$, O.A.Mojiminyi ${ }^{\text {b }}$, N. Salim ${ }^{\text {a }}$, \\ K. Al-Hajri ${ }^{\text {a }}$ \\ ${ }^{\mathrm{a} M i n i s t r y}$ of Health and ${ }^{\mathrm{b}}$ Department of Pathology, \\ Faculty of Medicine, Kuwait University, Kuwait
}

Introduction: Body mass index (BMI) is a widely used index of obesity; however, cutoffs for the definition and classification of obesity in different ethnic populations have been questioned. Bioelectrical impedance analysis (BIA) is a safe, accurate and inexpensive method that could be widely used to assess the degree of adiposity. This study evaluates and compares routine anthropometric measurements and the usefulness of BIA for the determination of the prevalence of overweight and obesity in young adults. Methods: 127 medical students (70 female and 57 male) aged 17-25 years were recruited in this cross-sectional study. We measured weight, height, BMI and waist/hip circumferences. Body fat percent $(\mathrm{BF} \%)$ was measured using BIA. BMI $>25$ and $>30$ were used as criteria for determining overweight and obese subjects, respectively. Results: The prevalences of obesity (BMI $>30$ ) among males and females were 22.8 and $10 \%$, respectively; the prevalences of central obesity (WC $>102 \mathrm{~cm}$ in males; WC $>80$ $\mathrm{cm}$ in females) were 21 and $10 \%$, respectively. Cutoffs (upper $95 \%$ CI) for anthropometric indices for the identification of obesity differed in males and females. In males with $\mathrm{BMI}>30$, upper $95 \%$ $\mathrm{CI}$ cutoffs were: $\mathrm{WC}=115 \mathrm{~cm}, \mathrm{WHR}=0.93$, and $\mathrm{BF} \%=34.5$; in females, the upper $95 \%$ CI values were: $\mathrm{WC}=116 \mathrm{~cm}$, WHR $=$ 0.84 , and $\mathrm{BF} \%=48.5$. Pearson correlation analysis showed that $\mathrm{BMI}(\mathrm{r}=0.68)$ showed the best correlation with $\mathrm{BF} \%$ compared to WC $(r=0.41)$ and WHR $(r=-0.027)$. WC $(r=0.85)$ showed a better correlation with BMI than WHR $(r=0.37)$. Conclusions: Obesity is prevalent among medical students. Despite its limitations, BMI showed the best correlation with BIA, and WHR showed the worst correlation. The cutoff values identified in this population are different from international guidelines. There is need to adjust the cutoff levels for the definition of obesity in the Kuwaiti population. BIA is simple, easy to perform and recommended for the routine determination of adipose tissue burden.

\section{Basic and Applied Sciences Awards}

\section{Loss of Estrogen Receptor in Human Breast Cancer Cells Is Associated with a Gradual Epithelial to Mesenchymal Transition

\author{
Y.A. Luqmani ${ }^{\text {a }}$, F. Al Mulla ${ }^{\text {b }}$, A. Al Azmi ${ }^{\text {C }}$, E. Tawfiq ${ }^{\text {d }}$, S. Al Saleh ${ }^{\mathrm{d}}$ \\ Faculties of apharmacy, ${ }^{\mathrm{b}}$ Medicine and ${ }^{\mathrm{C}}$ Science, and \\ ${ }^{\mathrm{d} C o l l e g e ~ o f ~ G r a d u a t e ~ S t u d i e s, ~ K u w a i t ~ U n i v e r s i t y, ~ K u w a i t ~}$
}

Introduction: Loss of functional estrogen receptor (ER) is central to the development of endocrine-resistant breast cancer. Subsequent therapeutic intervention would benefit from increased understanding of the associated molecular events participating in continued proliferation. Global gene expression was analysed in breast cancer cell lines that either overexpress ER (MCF7), are de novo ER-negative (MDA231) or in which ER is constitutively (pII) or inducibly (E2) downregulated by siRNA, in order to identify the transcriptional response to ER blockade. Methods: Labeled cRNA transcribed from cDNA synthesised from extracted cellular RNA was hybridised to replicate low- and high-density gene microarrays to compare phenotypic profiles of these cell lines; chemiluminescence/fluorescence signals were quantified with appropriate software packages. Selected differentially expressed genes were analysed by real-time quantitative PCR. Results: Lowdensity-array scanning highlighted several genes that discriminated MCF7 from MDA231 and pII; this was confirmed and extended in the high-density scans. pII cells exhibited elevated transcripts encoding proteins with motility functions, most crucially metastasis, such as urokinase plasminogen activator. Reduced ER expression was associated with loss of epithelial markers such as 
keratin 18/19 and increased appearance of transcripts of genes typically found in cells of mesenchymal origin: vimentin, fibronectin, cadherin 1, vascular endothelial growth factor and CD68. Differential expression of these genes was confirmed by PCR analysis. Tetracycline-induced transient ER downregulation in E2 cells failed to elicit the changes apparent in pII cells. Conclusions: Our observations suggest that a change from an epithelial to a more invasive fibroblastic phenotype may be concurrent with gradual adaptation to loss of the functional capacity of the ER transcriptional pathway, leading to chemoresistant estrogenindependent cancer.

\section{2}

\section{Effects of Omeprazole Treatment on Nucleoside Transporter Expression and Adenosine Uptake in the Rat Gastric Mucosa}

\section{Z. Redzic, F. Hasan, H. Al-Sarraf}

Departments of Physiology and Medicine, Faculty of Medicine, Kuwait University, Kuwait

Introduction: An increase in adenosine concentration has been shown to inhibit gastric acid secretion in the rat via adenosine receptors. This study aimed to examine the effects of omeprazole-induced achlorhydria on the expression and functional activity of nucleoside transporters in rat gastric mucosa. Methods: Wistar rats were treated for either 1 or 3 days with $0.4 \mathrm{mmol} / \mathrm{kg}$ omeprazole via gavage; controls were treated with the vehicle. The expression of nucleoside transporters at the transcript level and at the protein level was explored by quantitative real-time PCR assays and by immunoblotting, respectively. The functional activity of nucleoside transporters was explored by observing $\left[{ }^{3} \mathrm{H}\right]$ adenosine uptake in vitro. Results: Corporeal mucosa expressed rat equilibrative nucleoside transporters (rENT) 1 and 2, and rat concentrative nucleoside transporter ( $\mathrm{rCNT}) 1,2$ and 3 at the transcript level. Immunoblots revealed the presence of rENT1, rENT2, rCNT1 and $\mathrm{rCNT} 3$ at the protein level. In vitro uptake of $\left[{ }^{3} \mathrm{H}\right]$ adenosine by gastric mucosa consisted of $\mathrm{Na}^{+}$-dependent and $\mathrm{Na}^{+}$-independent components. One-day omeprazole treatment caused no changes in nucleoside transporter mRNA levels or in $\left[{ }^{3} \mathrm{H}\right]$ adenosine uptake. Three-day omeprazole treatments led to a 12-fold and 17-fold increase in rENT2 and rCNT1 mRNA levels, respectively; samples taken after 3-day treatment also took up significantly more $\left[{ }^{3} \mathrm{H}\right]$ adenosine than samples from the corresponding control. Conclusions: The observed changes in rENT2 and rCNT1 mRNA amounts as well as an increase in $\left[{ }^{3} \mathrm{H}\right]$ adenosine in vitro uptake in rat gastric mucosa after omeprazole treatment could cause a reduction in extracellular concentrations of that nucleoside. Modification of nucleoside transport by changes in intraluminal acidity may represent a novel regulatory feedback mechanism to control gastric acid secretion.

\section{Clinical Sciences Awards}

1

Baseline Characteristics, Management Practices and In-Hospital Outcome of Patients Hospitalized with Acute Coronary Syndromes in the Gulf (Gulf Registry of Acute Coronary Events)

M. Zubaid ${ }^{\mathrm{a}}$, W. Rashed ${ }^{\mathrm{b}}$, W. Almahmeed ${ }^{\mathrm{c}}$, J. Al-Lawati ${ }^{\mathrm{d}}$, K. Sulaiman ${ }^{\mathrm{e}}$, A. Al-Motarreb ${ }^{\mathrm{f}}$, H. Amin ${ }^{\mathrm{g}}$, J. Al Suwaidi ${ }^{\text {h }}$

${ }^{a}$ Faculty of Medicine, Kuwait University, and bepartment of Medicine, Mubarak Al-Kabeer Hospital, Ministry of Health, Kuwait; 'Sheikh Khalifa Medical City, United Arab Emirates; ${ }^{\mathrm{d}}$ Department of Non-Communicable Diseases Control, Ministry of Health, and ${ }^{\mathrm{E}}$ Royal Hospital, Oman; ${ }^{\mathrm{f}}$ Department of Medicine, Faculty of Medicine, Sana'a University, Yemen; ${ }^{9}$ Mohammed Bin Khalifa Cardiac Centre, Bahrain; hHamad General Hospital and Hamad Medical Corporation, Qatar

Introduction: Acute coronary syndrome (ACS) is a leading cause of morbidity and mortality in Gulf countries. Our aim was to describe the epidemiology, treatment and in-hospital outcomes of patients with ACS in this region. Methods: The Gulf Registry of Acute Coronary Events (Gulf RACE) is a prospective multinational multi-center survey of consecutive patients hospitalized with a final diagnosis of ACS over a period of 5 months (from February to June 2007). Results: A total of 6,706 ACS patients were recruited. The mean age was 56 years, and $76 \%$ were men. Half of the patients had a history of hypertension, while $41 \%$ were diabetics and $41 \%$ were smokers. The majority (78\%) were Killip class I at presentation. The final diagnosis was ST segment elevation myocardial infarction (STEMI) in 39\%, non-STEMI in 32\%, and unstable angina in 29\%. Among patients with STEMI, reperfusion therapy was administered to $63 \%$. Primary percutaneous coronary intervention (PCI) was performed in $6 \%$ of these patients. The median door-to-needle time was $40 \mathrm{~min}$, and the median door-to-balloon time was $79 \mathrm{~min}$. Coronary angiography during hospitalization, other than for primary PCI, was performed in $19 \%$ of patients. At discharge, aspirin was prescribed in $97 \%$ of patients, clopidogrel in $51 \%$, $\beta$-blockers in $78 \%$, angiotensin-converting enzyme inhibitors/angiotensin receptor blockers in $79 \%$, and statins in $83 \%$. The in-hospital mortality rate was $3.8 \%$. Conclusions: This is one of very few ACS registries relating to patients from the Middle East. It provides an insight into a different patient population and practice patterns compared to those of Europe and North America. Our patients are about a decade younger and have more diabetes as a risk factor. The reperfusion strategy used in the majority of patients is thrombolytic therapy, and the use of in-hospital cardiac catheterization is low. 


\section{2}

Evaluation of Adiponectin as a Potential Component of the Criteria for Metabolic Syndrome

N.A. Abdella a , O.A. Mojiminiyi ${ }^{\text {b }, ~ F . ~ A l-M u l l a ~}{ }^{\text {b }}$, H. Al-Mohammedic,

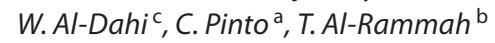

Departments of ${ }^{a}$ Medicine and ${ }^{b}$ Pathology, Faculty of Medicine, Kuwait University, and 'Department of Medicine, Mubarak Al-Kabeer Hospital, Kuwait

Introduction: First-degree relatives (FDR) of patients with type 2 diabetes (T2DM) have an increased risk of developing diabetes because of aggregation of cardiometabolic risk factors. This study explores the potential use of adiponectin as a marker of metabolic syndrome (MS) in normoglycemic FDR of T2DM patients. Methods: Fasting adiponectin, insulin, glucose, and full lipid profile were determined in 423 FDR and 53 healthy control subjects without a family history of diabetes. Clinical and anthropometric data were recorded, and subjects were classified on the basis of the degree of adiposity, insulin resistance (homeostasis model assessment of insulin resistance; HOMA-IR) and the number of criteria of MS (International Diabetes Federation). Results: Adiponectin concentration was higher in females than males (mean 9.7 vs. $6.9 \mu \mathrm{g} / \mathrm{ml}$ ), despite similar waist circumferences (WC). In both FDR and controls, adiponectin was inversely correlated with WC and HOMA-IR and positively correlated with HDL cholesterol. Adiponectin showed a stepwise decrease with an increasing number of MS criteria. Binary logistic regression showed that the odds ratio of MS as predicted by adiponectin was 0.55 (95\% CI = 0. 41-0.73; p < 0.0001). At a cutoff point of $7.5 \mu \mathrm{g} /$ $\mathrm{ml}$, the diagnostic sensitivity and specificity of adiponectin for MS were 90 and $70 \%$, respectively, compared to 42 and $95 \%$ for triglycerides and 80 and 54\% for HDL cholesterol at standard cutoff points. Receiver operating characteristic analysis showed that adiponectin (0.859) had a significantly larger area under the curve compared with HDLC (0.745) and triglycerides (0.823) for detection of MS. Conclusions: Adiponectin should be an additional and useful criterion for identification of MS.

\section{3}

\section{Putative Roles of Circulating Resistin in Patients with Asthma or COPD and Cigarette Smokers}

\section{S.S. Al Mutairi ${ }^{\text {a }, ~ O . A . ~ M o j i m i n i y i ~}{ }^{\text {b }}$, A. Shihab-Eldeen ${ }^{c}$, T. Al Rammah ${ }^{\text {b }, ~ N . A . ~ A b d e l l a ~}{ }^{\text {a }}$}

Departments of a Medicine, bPathology and 'Pharmacology, Faculty of Medicine, Kuwait University, Kuwait

Introduction: Resistin has been linked to several inflammation-related diseases like atherosclerosis, arthritis and recently to inflammatory lung diseases. The main aim of this study was to explore the associations and putative roles of resistin in patients with acute and stable inflammatory obstructive airway diseases and cigarette smokers. Methods: We determined fasting resistin, complements C3 and C4, insulin, glucose and lipid profiles, and calculated insulin resistance (homeostasis model assessment; HOMA-IR) in patients with acute asthma exacerbation $(\mathrm{n}=34)$, stable asthma $(\mathrm{n}=26)$ and COPD $(\mathrm{n}=26)$, cigarette smokers $(\mathrm{n}=$ $81)$, and healthy control subjects $(n=42)$. Uni- and multivariate regression analyses were used to find the associations between these variables and pulmonary function tests. Results: Patients with COPD or asthma (acute or stable) had significantly higher resistin and insulin levels than control subjects. Resistin ( $\mathrm{p}=$ $0.019)$, insulin ( $p=0.03)$, HOMA-IR $(p=0.02)$, FEV $_{1}(p=0.002)$ and $\mathrm{FEV}_{1} / \mathrm{FVC}(\mathrm{p}<0.0001)$ were significantly different when patients with acute asthma were compared with stable asthma and COPD; smokers had similar levels of resistin, C3 and C4 as patients with asthma and COPD, which were significantly higher than in control subjects. In smokers and patients with asthma or COPD, resistin showed significant inverse correlations with $\mathrm{FEV}_{1}$ and $\mathrm{FEV}_{1} / \mathrm{FVC}$, and positive significant correlations with BMI and HOMA-IR. Logistic regression analyses showed that resistin is associated with inflammatory obstructive airways disease $(\mathrm{OR}=1.22 ; \mathrm{p}=0.001)$ and smoking $(\mathrm{OR}=1.18 ; \mathrm{p}<0.0001)$. Conclusions: Smoking and conditions with pulmonary inflammation are accompanied by higher resistin, which contributes to an insulin-resistant state. The higher resistin in smokers is independent of the BMI and the degree of insulin resistance and may be a key component linking smoking, insulin resistance and cardiovascular disease.

\section{Case Report Award}

\section{Case of a King Cobra Bite in Kuwait: Ethical, Public Health, and Legal Issues}

M. Al Tarrah ${ }^{\text {a }}$ M. Al Asfoor ${ }^{\text {a }}$, K. Abdul Malek ${ }^{a}$, M. Bouhaimed ${ }^{\text {b, } c}$

antensive Care Unit, Al Amiri Hospital, Ministry of Health, and Departments of ${ }^{\mathrm{b}} \mathrm{Community}$ Medicine and 'Surgery, Faculty of Medicine, Kuwait University, Kuwait

Background: The king cobra is the longest venomous snake in the world. The venom of this snake is neurotoxic, composed mostly of proteins and polypeptides, and it can kill humans in a single bite. The mortality rate can be as high as $75 \%$. The growing trend toward the collection of exotic snakes by private collectors increases the likelihood that emergency doctors will face the challenge of treating such cases. Case Summary: A 26-year-old Kuwaiti gentleman presented to a hospital casualty department after being bitten by 'a snake'. The patient went into complete respiratory arrest and was intubated and shifted to the intensive care unit. He received multiple doses of polyvalent antivenom for snakes indigenous to Kuwait with no effect. After initially giving misleading information, detailed questioning of the family members revealed that a smuggled highly toxic king cobra was the culprit, and that the patient owned more than 180 snakes at his home in a residential area in Kuwait. Locating and bringing the spe- 
cific antivenom from Thailand was laborious and time-consuming. Within $30 \mathrm{~min}$ of administering the antivenom, the patient's total paralytic status was reversed. The patient was successfully extubated and discharged to the general floor the next day. This case raises numerous issues that need careful evaluation and analysis: (1) the consequences of hiding information or misleading the medical team; (2) the patient did not have the necessary permits to possess these snakes in Kuwait; (3) one of the patient's employees had been admitted with a snake bite to the same hospital 2 months earlier, but had not disclosed details of his work environment; (4) the conflict between protecting confidentiality and the duty to report any identified public health hazards. Conclusion: This is the first reported case of a king cobra bite in $\mathrm{Ku}$ wait, and it presents challenging medical, ethical, legal and public health/safety issues. 